\title{
ADENOCARCINOMA PRIMÁRIO DE DUODENO
}

\author{
ADENOCARCINOMA OF DUODENUM
}

\author{
Fernando Costa Nunes, TCBC-MG ${ }^{1}$ \\ Alcino Lázaro da Silva, TCBC-MG ${ }^{2}$
}

\section{INTRODUÇÃO}

O intestino delgado, apesar de sua extensão, é local infreqüente de neoplasias, abrigando cerca de $2 \%$ dos tumores do tubo gastrointestinal. O tipo histológico prevalente é o adenocarcinoma, seguido dos tumores carcinóides.

$\mathrm{O}$ adenocarcinoma do intestino delgado foi inicialmente descrito por Hamberger, em 1746. Há pouco mais de 2.500 casos relatados na literatura até esta data. Ocorre com mais freqüência no sexo masculino, na proporção de $2: 1$, principalmente na sétima década de vida e é mais freqüente no duodeno, seguido do jejuno e íleo ${ }^{1,2}$.

O diagnóstico precoce é dificultado pela ausência de sintomas específicos e pelos conhecimentos restritos sobre esta entidade rara. Exames como a radiografia contrastada do esôfago, estômago e duodeno, esofagogastroduodenoscopia com biopsia, ultra-sonografia e tomografia computadorizada do abdome podem auxiliar no diagnóstico. Duodenopancreatectomia, pela técnica de Whipple, é a melhor opção terapêutica na localização duodenal. ${ }^{3}$

A escassez de relatos de portadores dessa doença e a sua associação com polipose satélite, submetidos à cirurgia curativa, nos estimulou à apresentação do caso.

\section{RELATO DO CASO}

J. O. C., 61a, masculino, branco, casado, lavrador, admitido no HCFMIT com história de anorexia e vômitos há seis meses, acompanhados de dor abdominal pós-prandial, tipo cólica, de moderada intensidade, na região epigástrica.

Os vômitos eram escuros, do tipo borra de café e, posteriormente, biliosos. Nega pirose, hematêmese, melena ou alterações do hábito intestinal. Perda de $8 \mathrm{~kg}$ desde o início dos sintomas. Ao exame físico, encontrava-se em regular estado geral, com mucosas secas e com dor de moderada intensidade, à palpação profunda, no epigástrio. Exames de rotina, normais. CEA 0,94, AFP0,61.

Trânsito intestinal mostrava distensão e estreitamento que se fazia de maneira abrupta, localizado no quarto segmento do duodeno, com perda do pregueado mucoso (Figura 1).

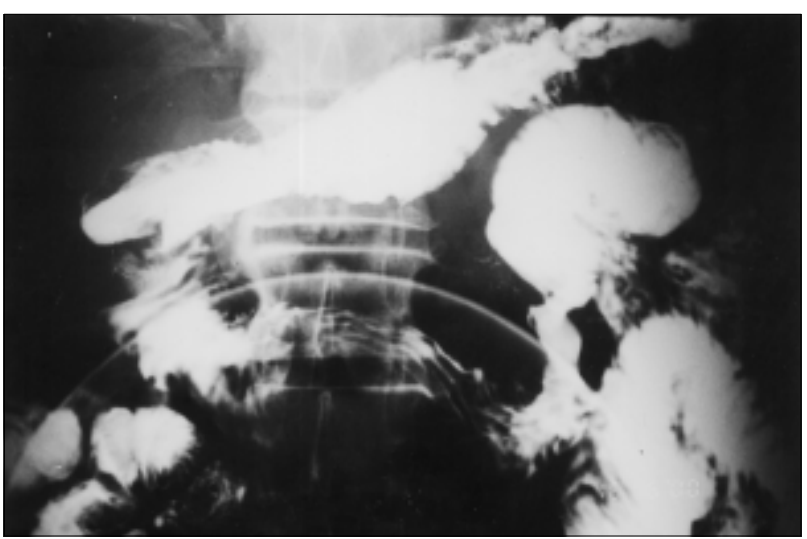

Figura 1 - Radiografia do trânsito intestinal mostrando distenção e estenose abrupta na $4^{a}$ porção do duodeno.

Ultra-som do abdome revelava fígado, vesícula biliar, pâncreas,baço e rins sem alterações. Espessamento da parede duodenal em topografia da sua $4^{\mathrm{a}}$ porção. Aorta abdominal com calibre próximo ao limite da normalidade, discretamente tortuosa. Tomografia computadorizada do abdome superior demonstrava segmento de alça do delgado com espessamento de paredes em topografia da região epigástrica.

Operado no dia 16-06-2000, foi realizada enterectomia parcial com retirada da $4^{\text {a }}$ porção duodenal e $10 \mathrm{~cm}$ de jejuno.

1. Professor adjunto do departamento de Cirurgia Geral da Faculdade de Medicina de Itajubá; Mestre em Cirurgia — UFMG

2. Professor Titular de Cirurgia do Aparelho Digestivo — UFMG

Recebido em 23/10/2000

Aceito para publicação em 26/03/2002

Trabalho da Pós-Graduação em Cirurgia da Universidade Federal de Minas Gerais e do Departamento de Cirurgia da Faculdade de Medicina de Itajubá 
O exame anatomopatológico confirmou o tumor de $4^{\mathrm{a}}$ porção do duodeno, do tipo adenocarcinoma anular moderadamente diferenciado, infiltrando até a camada muscular. Ausência de metástases em todos os linfonodos examinados: T3N0M0 (Figuras 2 e 3).

Quando a peça cirúrgica foi aberta, para estudo, no Departamento de Anatomia Patológica, encontramos três pólipos vilosos, de base larga $(2,0 \times 1,7 \times 1,5 \mathrm{~cm} ; 3,0 \times 1,0 \times 1,0$ $\mathrm{cm}$ e 2,0x1,0x0,7 cm ), no local da estenose, os quais estavam ocluindo a luz intestinal e apresentavam acometimento tumoral discreto em sua base.

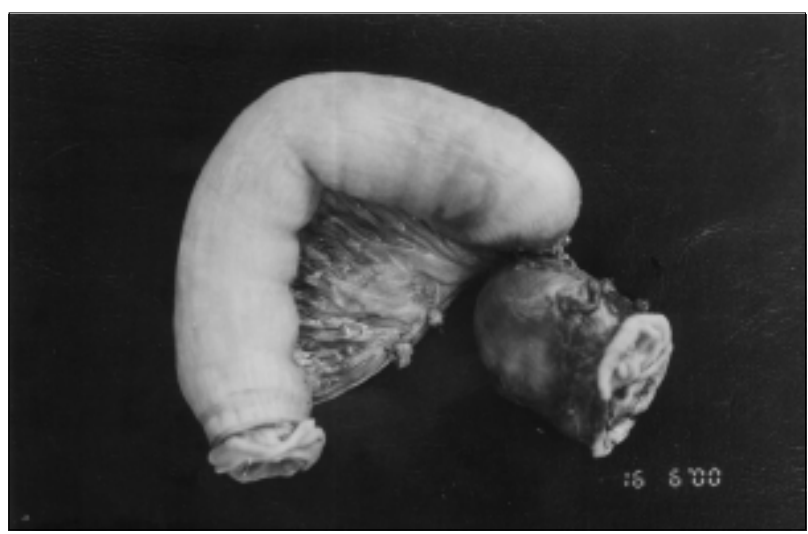

Figura 2 - Espécime de enterectomia.

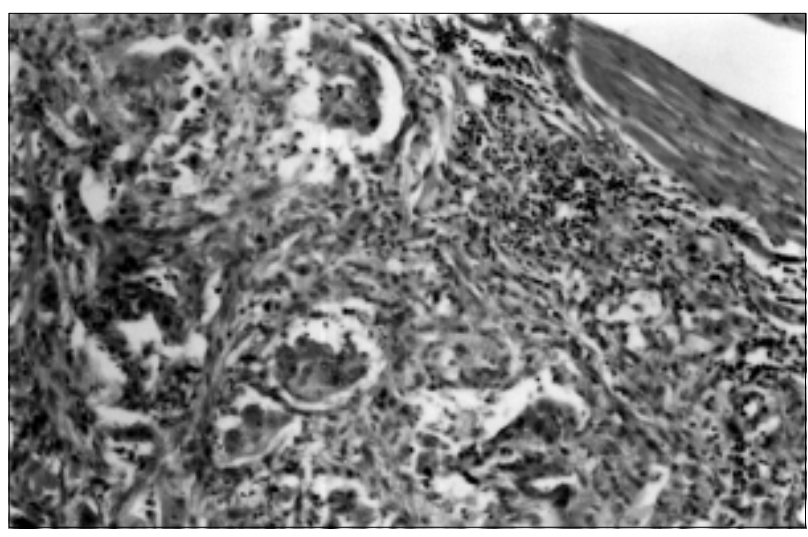

Figura 3 - Fotomicrografia do duodeno evidenciando o tumor invadindo a camada muscular (HE x100).

\section{DISCUSSÃO}

Tumores do intestino delgado são mais freqüentes em homens e na etnia branca. Noventa por cento dos casos ocorrem acima dos 40 anos, sendo a média de idade $60 \operatorname{anos}^{3}$. Os sintomas são vagos e inespecíficos, com dor abdominal, náuseas, vômitos intermitentes, anorexia e distensão abdominal pós-prandial, contribuindo para um diagnóstico tardio e prognóstico reservado. Anemia pode ser notada, assim como icterícia e anormalidades dos testes de função hepática. Quanto à localização, há uma predominância da segunda porção duodeno (40\%), seguida da terceira e quarta (45\%), e primeira porção $(15 \%)^{1,2}$. Em relação aos exames complementares, tanto a radiografia contrastada do esôfago, estômago e duodeno como a endoscopia digestiva alta com biopsias, podem ser realizadas como métodos diagnósticos iniciais. A tomografia computadorizada do abdome pode detectar aproximadamente metade dessas lesões, além de evidenciar a presença de linfoadenomegalias. A ultra-sonografia endoscópica tem sido recentementemente utilizada com o intuito de determinar o estágio do tumor ${ }^{2}$.

Duodenopancreatectomia pela técnica de Whipple é o procedimento de escolha para o tratamento dessas neoplasias, uma vez que permite a ressecção em bloco com os linfonodos regionais, porém, apenas $30 \%$ destes tumores são ressecáveis. Está indicada, também, como tratamento curativo, para os pacientes com linfonodos comprometidos. A sobrevida, em cinco anos, para os pacientes submetidos a esse procedimento corresponde a $40 \%{ }^{2}$.

A ressecção segmentar pode ser aplicada, com intenção curativa, para tumores pequenos da terceira e, especialmente, da quarta porção do duodeno, desde que não exista comprometimento linfonodal e uma invasão em profundidade limitada ${ }^{1,2,4}$, o que ao nosso ver aconteceu neste caso.

A gastrojejunostomia é realizada naqueles pacientes que apresentam doença irressecável no ato operatório ${ }^{2}$. A papiloduodenectomia, técnica cirúrgica aplicada para casos selecionados de tumores da papila de Vater, não tem lugar para as neoplasias primárias de duodeno. A linfadenectomia pode ser incluída entre as opções terapêuticas em tumores malignos de intestino delgado $^{5}$. O tratamento adjuvante com quimioterapia e radioterapia deve ser individualizado, já que não há evidências convincentes de que possam trazer benefícios à sobrevida ${ }^{1,2}$.

A maioria dos estudos relata uma sobrevida pequena para adenocarcinoma do intestino delgado, embora a literatura seja contraditória a este respeito. A sobrevida em cinco e dez anos é de $39 \%$ e $37 \%$, respectivamente, para as neoplasias primárias do duodeno ressecáveis e correlaciona-se com a fase do diagnóstico. Os pacientes submetidos à cirurgia paliativa têm sobrevida de meses. $\mathrm{O}$ tumor anular circunferencial, mais comumente visto no adenocarcinoma, expande-se e vagarosamente oclui o lúmen. Quanto maior a lesão mais precocemente surgem os sintomas de náuseas e vômitos. As características clínicas do caso relatado foram semelhantes às referidas na literatura, especialmente quanto à sintomatologia inicial, à faixa etária acometida e à localização do tumor no duodeno. A investigação diagnóstica e o tratamento adotado foram semelhantes aos da maioria dos estudos encontrados na literatura.

A precocidade do diagnóstico ocorreu por tratar-se de um caso de semi-oclusão intestinal alta pois, no local acometido pelo adenocarcinoma anular, havia três pólipos sésseis que também estavam acometidos pelo tumor, na sua base. 


\begin{abstract}
Primary adenocarcinoma of the duodenum is an extremely rare disease, and represents only $0.35 \%$ of all gastrointestinal malignancies. Early detection of the disease may be difficult because of the absence of pathognomonic symptoms. The authors relate one case of a adenocarcinoma of the duodenum in a 61-year-old white man with a history of abdominal pain for a six-month period, associated with postprandial fullness, vomiting and weight loss. Contrasted $x$-ray and computerized tomography showed a tumor in the fourth segment of the duodenum, with partial obstruction of the lumen. Histological study revealed a moderate differentiated adenocarcinoma. Treatment consisted of resection of the fourth portion of duodenum. The authors comment on the most important aspects of this pathology.
\end{abstract}

Key Words: Duodenum; Cancer of the duodenum; Adenocarcinoma of the duodenum; Adenocarcinoma of the small bowel.

\title{
REFERÊNCIAS
}

1. Scott-Coombes DM, Williamson RCN. Surgical treatment of primary duodenal carcinoma a personal series. British Journal of Surgery 1994, 81:1472-4.

2. Pickleman J, Koelsch M, Cheifec G. Node-positive duodenal carcinoma is curable. Arch Surg 1997; 132:241-4.

3. Chow JS, Chen CC, Ahsan H, et al. A population-based study of the incidence of malignant small bowel tumours: SEER, 1973-1990. International Journal of Epidemiology 1996; 25: 722-8.

4. Bromberg SH, Goffi PS, Goffi, FS et al. Neoplasias Duodenais Primárias: Anàlise de casuística e conduta cirúrgica. Rev Col Bras Cir 2000; 27: 267-0.
5. Lázaro da Silva A, Galizzi J, Conceição AS. Linfoadenectomia mesentérica no linfossarcoma do intestino delgado. Rer. Ass. Méd Minas Gerais 977; 29 (3):92-93.

Endereço para correspondência:

Dr. Fernando Costa Nunes

Rua Miguel Mohallem, 193

37500-000 — Itajubá-MG 